\title{
Composition at the "ankle" measured by the Pierre Auger Observatory: pure or mixed?
}

\author{
Alexey Yushkov ${ }^{*, a}$ for the Pierre Auger Collaboration ${ }^{b}$ \\ ${ }^{a}$ University of Siegen, Department of Physics, 57072, Siegen, Germany \\ ${ }^{b}$ Observatorio Pierre Auger, Av. San Martín Norte 304, 5613 Malargüe, Argentina \\ E-mail: auger_spokespersons@fnal.gov \\ Full author list: http://www.auger.org/archive/authors_2015_06.html
}

\begin{abstract}
We report for the first time on the measurement of the correlation between the depth of shower maximum and the signal in water-Cherenkov stations for events reconstructed by both the fluorescence and the surface detectors of the Pierre Auger Observatory. Such a correlated measurement is a unique feature of a hybrid air-shower observatory and allows us to determine the purity of the cosmic-ray composition. The observed correlation in the energy range around the "ankle" $\lg (E / \mathrm{eV})=18.5-19.0$ differs significantly from the expectations for pure beams, indicating that the primary composition in this range is mixed, unless the hadronic interactions at these energies behave very differently than in conventional, LHC-tuned event generators.
\end{abstract}

The 34th International Cosmic Ray Conference,

30 July- 6 August, 2015

The Hague, The Netherlands

${ }^{*}$ Speaker. 


\section{Introduction}

The determination of the mass composition of ultra-high-energy cosmic rays (UHECRs) is one of the most challenging problems for extensive air-shower (EAS) experiments; for a recent review, see [1]. Parameters of hadronic interactions at these energies are only loosely constrained by accelerator data and thus the evolution of the behavior of EAS properties with energy can in general be interpreted in terms of both changes of the primary mass and/or of the characteristics of the particle interactions. One way to try to resolve this ambiguity is to find shower parameters or their combinations that rely on more general aspects of EAS physics and are thus relatively insensitive to the uncertainties in the properties of the hadronic interactions.

In particular, in [2] it was proposed to use the correlation between the depth of the shower maximum $X_{\max }$ and the number of muons $N_{\mu}$ of the EAS for the determination of the degree of purity of the beam, i.e., whether it is composed of several or just one nuclear species. In the present work we adapt this idea to the conditions of the Pierre Auger Observatory [3]. In place of $N_{\mu}$ we use the total signal in water-Cherenkov detectors at 1000 meters from the core, $S(1000)$, a substantial fraction of which is due to muons: from $40 \%$ to $90 \%$ for zenith angles from $20^{\circ}$ to $60^{\circ}$ [4]. We show that the correlation $\left(X_{\max }, S(1000)\right)$ for pure primary beams for all current interaction models turns out to be close to zero or positive, while for well-mixed compositions with a large spread of masses it becomes negative (see [2]). Thus the correlation coefficient can be used to determine the dispersion, $\sigma(\ln A)$, of primary masses, given by $\sigma(\ln A)=\sqrt{\left\langle\ln ^{2} A\right\rangle-\langle\ln A\rangle^{2}}$ where $\langle\ln A\rangle=\sum_{i} f_{i} \ln A_{i}$ and $\left\langle\ln ^{2} A\right\rangle=\sum_{i} f_{i} \ln ^{2} A_{i}$ with $f_{i}$ being the relative fraction of mass $A_{i}$.

An estimation of the degree of purity of the primary beam in the energy range $\lg (E / \mathrm{eV})=$ $18.5-19.0$ is of particular interest as a test of the 'dip' scenario [5]. In this scenario the break in the energy spectrum at around $\lg (E / \mathrm{eV})=18.7$ results from electron-positron pair-production by extragalactic protons interacting with the cosmic microwave background. The 'dip' is well pronounced only if the fraction of heavier nuclei at the acceleration site is $\lesssim 15 \%$ (see [6] and references therein).

\section{Data and simulations}

The analysis is based on the same hybrid events as used in [7] recorded by both fluorescence (FD) and surface detectors (SD) of the Pierre Auger Observatory during the period from 01.01.2004 to 31.12.2012. The procedure of data selection, described in [7], guarantees that only high quality events are included in the analysis and that the mass composition of the selected sample is unbiased. The use of the signal in ground stations requires an additional application of the fiducial trigger cut [8] (the station with the highest signal should have at least 5 working neighbour stations), and exclusion of events with stations having saturated signal traces. The final data set for energies $\lg (E / \mathrm{eV})=18.5-19.0$ and zenith angles $\theta=0^{\circ}-65^{\circ}$ contains 1376 events.

Monte-Carlo (MC) simulations are performed with CORSIKA [9] for high-energy interaction models QGSJetII-04 [10], Epos-LHC[11] and Sibyll 2.1 [12]. FLUKA [13] is used to treat low energy interactions. For CORSIKA events, full detector simulation and reconstruction procedures

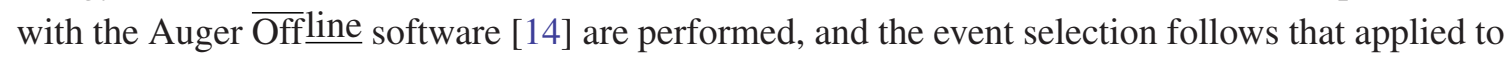



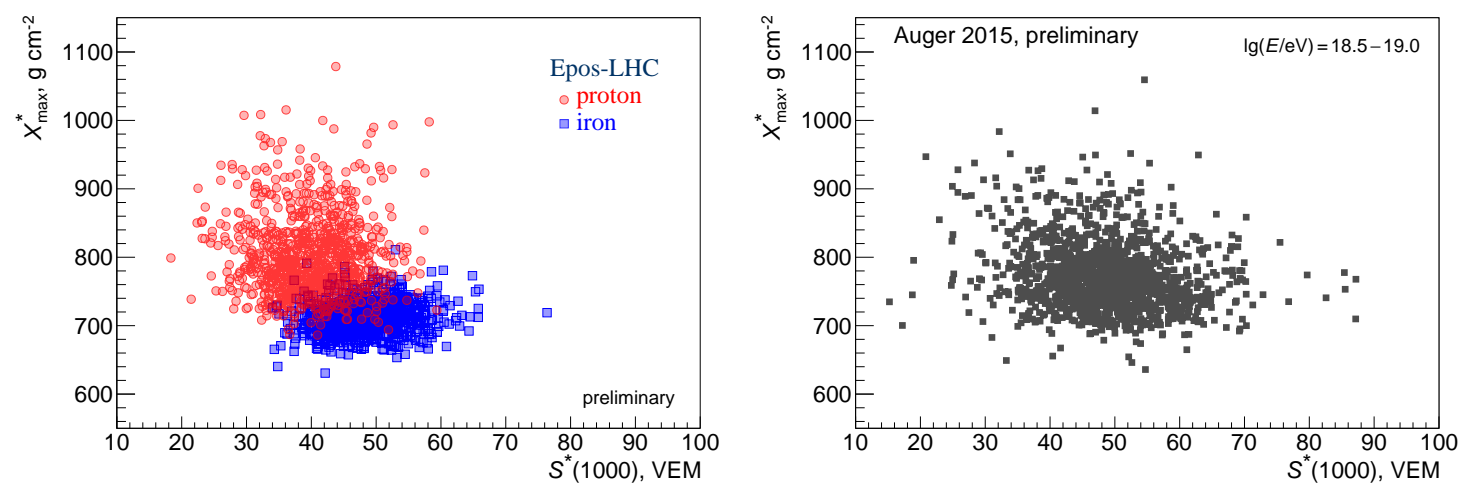

Figure 1: Scatter plot of $X_{\max }^{*}$ vs $S^{*}(1000)$ for protons and iron of Epos-LHC from full detector simulations (left) and for data (right) for $\lg (E / \mathrm{eV})=18.5-19.0$.

data. After selection, the proton samples for all models contain $\simeq 10^{4}$ showers; for heavier nuclei the samples vary from $5 \cdot 10^{3}$ to $10^{4}$ showers.

Since $S(1000)$ and $X_{\max }$ of an air shower depend on the energy and, in the case of $S(1000)$, also on the zenith angle, we scale $S(1000)$ and $X_{\max }$ to a reference energy and zenith angle. In this way, a decorrelation between the observables from combining different energies and zenith angles in the data set is avoided. We scale $S(1000)$ to $38^{\circ}$ and $10 \mathrm{EeV}$ using the calibration curves from [15] and $X_{\max }$ to $10 \mathrm{EeV}$ using an elongation rate of $58 \mathrm{~g} \mathrm{~cm}^{-2} / \mathrm{decade}$. These scaled quantities will be marked with an asterisk: $X_{\max }^{*}, S^{*}(1000)$. Fig. 1 (right panel) shows the correlation between $X_{\max }^{*}$ and $S^{*}(1000)$ observed in data. Also shown, for illustration purposes, are the simulations for proton and iron primaries with Epos-LHC.

\section{Method and results}

As a measure of the correlation between $X_{\max }^{*}$ and $S^{*}(1000)$ we take the ranking coefficient $r_{\mathrm{G}}$ introduced by Gideon and Hollister in [16]. All events are ranked in both $X_{\max }^{*}$ and $S^{*}(1000)$, and the measured values of these observables are replaced by ranks for calculating the correlation. Further, the values of ranks are not used directly to calculate $r_{\mathrm{G}}$, but rather the general statistical dependence between $X_{\max }^{*}$ and $S^{*}(1000)$ is estimated counting numbers of events with ranks deviating from the expectations for perfect correlation and anti-correlation. With respect to the classical Pearson and Spearman coefficients, $r_{\mathrm{G}}$ provides a more robust estimate of the correlation $[16,17]$. In particular, $r_{\mathrm{G}}$ is less sensitive to the removal of the most influential events or to outliers. We also note that the difference between correlation coefficients found in data and $\mathrm{MC}$ simulations for pure beams gets larger using Pearson and Spearman coefficients (or a number of other correlation coefficients considered in [17]) compared to using $r_{\mathrm{G}}$ so that the choice of $r_{\mathrm{G}}$ can be also viewed as conservative. The statistical uncertainty of $r_{\mathrm{G}}$ is determined using dedicated simulations and for the sample of size $N$ it is $\sigma_{\text {stat }}\left(r_{\mathrm{G}}\right) \approx 0.9 / \sqrt{N}$.

In Table 1 we present the $r_{\mathrm{G}}$ values for data and for the simulations for pure beams. Compared to data, where the correlation is significantly negative $r_{\mathrm{G}}\left(X_{\max }^{*}, S^{*}(1000)\right)=-0.125 \pm 0.024$ (stat), the smallest difference is found for Epos-LHC protons and it is around $5 \sigma_{\text {stat. }}$ Pre-LHC versions 
Table 1: $r_{\mathrm{G}}\left(X_{\max }^{*}, S^{*}(1000)\right)$ for data and for MC simulations of pure beams (preliminary). Statistical uncertainties on the MC values are $\sigma_{\text {stat }} \approx 0.01$.

\begin{tabular}{cccc}
\hline data & \multicolumn{3}{c}{$-0.125 \pm 0.024$ (stat) } \\
\hline \hline & Epos-LHC & QGSJetII-04 & Sibyll 2.1 \\
\hline$p$ & 0.00 & 0.08 & 0.07 \\
$\mathrm{He}$ & 0.08 & 0.15 & 0.15 \\
$\mathrm{O}$ & 0.09 & 0.15 & 0.14 \\
$\mathrm{Fe}$ & 0.08 & 0.12 & 0.12 \\
\hline
\end{tabular}
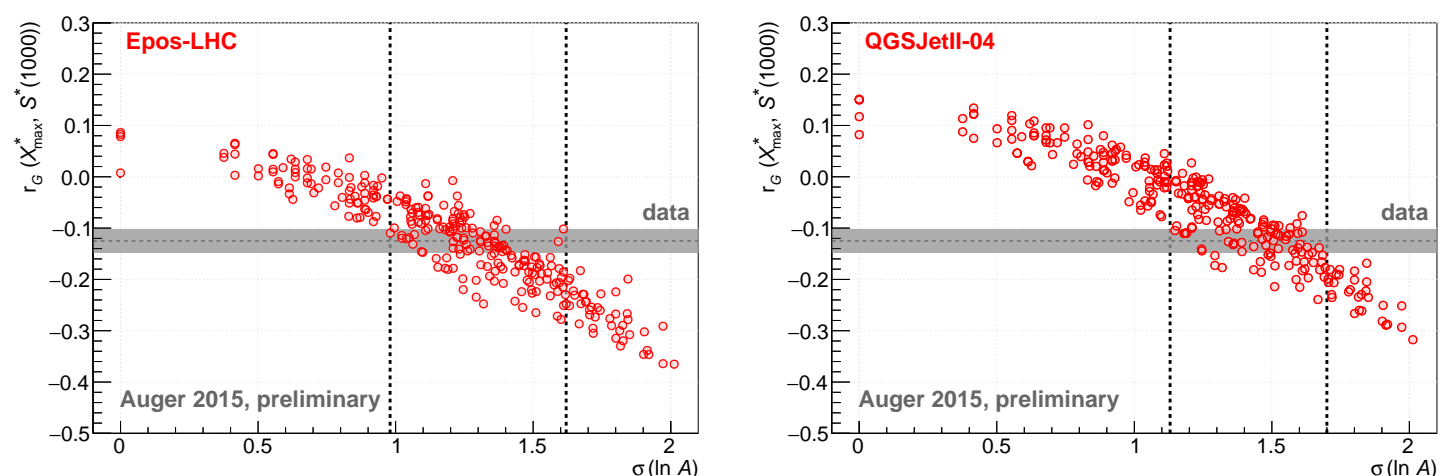

Figure 2: Dependence of the correlation coefficients $r_{\mathrm{G}}$ on $\sigma(\ln A)$ for Epos-LHC (left), QGSJetII-04 (right). Each MC point corresponds to a mixture with different fractions of protons, helium, oxygen and iron, the relative fractions change with 0.1 steps ( 4 points for pure beams are grouped at $\sigma(\ln A)=0$ ). The shaded band shows the $1 \sigma_{\text {stat }}$ interval for data. Vertical dotted lines indicate the range of $\sigma(\ln A)$ in simulations compatible with the observed correlation in data.

of Epos and QGSJetII produce values of correlations similar to Epos-LHC and QGSJetII-04. The differences between data and simulations are larger for pure beams other than protons. Using Pearson and Spearman coefficients one gets the same or slightly more positive values for pure beams as with $r_{\mathrm{G}}$, and more negative correlation for data: $r$ (Pearson) $=-0.210 \pm 0.038$ (stat); $r($ Spearman $)=-0.199 \pm 0.027$ (stat). This result shows that the composition in the considered energy range is not pure but mixed.

Fig. 2 presents the dependence of the correlation $r_{\mathrm{G}}\left(X_{\max }^{*}, S^{*}(1000)\right)$ on the dispersion of primary masses $\sigma(\ln A)$. Each MC point in this plot represents a mixture containing different fractions of protons, helium, oxygen and iron. The relative fractions $f_{i}$ of each species change with 0.1 steps between different mixtures. There are four points corresponding to beams of pure $p$, He, $\mathrm{O}$, and $\mathrm{Fe}$, grouped on the left side at $\sigma(\ln A)=0$; of these, the proton beam has the smallest $r_{\mathrm{G}}$ (cf. Table 1). The maximum possible value of $\sigma(\ln A) \simeq 2.01$ corresponds to the $0.5 p-0.5 \mathrm{Fe}$ mix.

The value of the correlation in data, indicated with the shaded band, is compatible with the MC samples with dispersions of primary masses $\sigma(\ln A) \gtrsim 1$. The conclusions on $\sigma(\ln A)$ are similar for all models considered (for Sibyll 2.1 one gets almost identical results to QGSJetII-04, 
cf. Table 1) and thus is weakly sensitive to the uncertainties in the description of the high-energy hadronic interactions.

The robustness of the presented approach makes it suitable for testing the self-consistency of the hadronic interaction models. For example, using the fractions of primary nuclei obtained from the fits of Auger $X_{\max }$ distributions [18] for QGSJetII-04 and Sibyll 2.1, which in the $\lg (E / \mathrm{eV})=$ $18.5-19.0$ energy range are close to $0.5 p-0.5 \mathrm{He}(\sigma(\ln A) \approx 0.7)$, one gets $r_{\mathrm{G}} \approx 0.07-0.09$. The incompatibility of this value with the results of the present correlation analysis may be an indication of deficiencies in these two interaction models. The composition found in [18] from $X_{\max }$ fits with Epos-LHC is close to $\approx 0.35 p-0.30 \mathrm{He}-0.35 \mathrm{O} \operatorname{mix}(\sigma(\ln A) \approx 1.17)$, and the corresponding correlation $r_{\mathrm{G}}=-0.084$ is within $2 \sigma$ from the $r_{\mathrm{G}}$ value in data.

\section{Uncertainties}

A number of standard tests were performed for estimation of the robustness of the obtained results. These checks include analysis of data recorded in various time periods and by different FD telescopes, separation of data in several angular ranges, and study of $r_{\mathrm{G}}$ in smaller energy bins. Results were consistent in all cases.

The ranking correlation coefficients are invariant with respect to any transformations not affecting ranks of the events. Thus $r_{\mathrm{G}}$ is insensitive to the systematic effects on $X_{\max }^{*}$ or $S^{*}(1000)$ that might lead to shift or multiplication of these observables by a constant value. In particular we have checked that the recent changes in Auger energy and $X_{\max }$ scales $[19,7]$ do not change the observed correlation. The same insensitivity of $r_{\mathrm{G}}$ was observed with respect to the application of various FD selection cuts which have been used in our publications from 2010 [20] until 2014 [7]. Finally, we introduced arbitrary energy and zenith angle dependent biases in $X_{\max }^{*}$ (up to $10 \mathrm{~g} \mathrm{~cm}^{-2}$ ) and $S^{*}(1000)$ (up to $10 \%$ ) and this changed $r_{\mathrm{G}}$ by $\simeq 0.01$. We take that value as a conservative estimate of the systematic error on $r_{\mathrm{G}}$.

We checked whether moderate changes of hadronic interaction parameters could make the value of $r_{\mathrm{G}}$ predicted for a pure proton composition consistent with observations. Using the approach described in [21] we performed simulations with Epos-LHC modifying the cross-section, multiplicity, elasticity and pion charge ratio in proton-air interactions by a factor $f_{19}=1.5$, i.e. increasing them by a factor linearly growing with $\lg E$ from 1.0 at $10^{15} \mathrm{eV}$ to 1.5 at $10^{19} \mathrm{eV}$ with respect to the nominal values [21]. CONEX [22] with 3D option was used for approximate estimation of the signal in Auger stations at 1000 meters from the core. It turned out that $r_{\mathrm{G}}$ is practically insensitive to the modifications of these interaction parameters decreasing only by $\Delta r_{\mathrm{G}} \lesssim 0.03$. The change in $r_{\mathrm{G}}$ due the increase of cross-section, still being small compared to the difference between data and pure proton expectations, is found to be zenith angle dependent and it would also lead to zenith angle dependent (and thus contradictory) conclusions on $\sigma(\ln A)$. Such a scenario is constrained additionally by other Auger findings (e.g. regarding the proton-air cross section derived for $\lg (E / \mathrm{eV})=17.8-18.5[23,24])$, making it implausible as an explanation of our observations.

A possible under-production of muons by the current interaction models [25, 26, 27] could lead to changes in the ordering of events in the $\left(X_{\max }^{*}, S^{*}(1000)\right)$ plane due to the presence of events with largely varying muon fractions of $S^{*}(1000)$. We performed a number of studies using CORSIKA

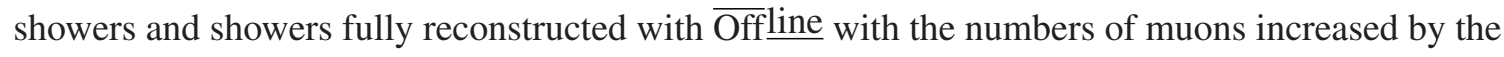


factors ranging from 1.1 to 1.9 and we have found that for the muon scaling by a factor $\approx 1.3$, as suggested by data for Epos-LHC $[25,26]$, the $r_{\mathrm{G}}$ value decreseas by $\lesssim 0.03$.

\section{Summary}

The observed correlation $r_{\mathrm{G}}\left(X_{\max }^{*}, S^{*}(1000)\right)$ between depth of shower maximum and total signal at 1000 meters from the core differs significantly from the correlations for any pure beam for simulations with Epos-LHC, QGSJetII-04 and Sibyll 2.1. The result is invariant with respect to additive and multiplicative scale transformations of the two variables and to any other transformations of $X_{\max }$ and $S(1000)$ which leave ranks of events unchanged, and hence is robust against many possible experimental systematic uncertainties. Several modifications of hadronic interactions were studied. The conclusions remain robust also with regard to hadronic uncertainties, unless hadronic interactions at these energies behave very differently than in conventional, LHC-tuned event generators. The results are compatible with a mixed primary composition around the 'ankle' with the dispersion of masses $1.0 \lesssim \sigma(\ln A) \lesssim 1.7$ and question the 'dip' scenario.

\section{References}

[1] K.-H. Kampert and M. Unger, Measurements of the Cosmic Ray Composition with Air Shower Experiments, Astropart.Phys. 35 (2012) 660-678, [1201. 0018 ].

[2] P. Younk and M. Risse, Sensitivity of the correlation between the depth of shower maximum and the muon shower size to the cosmic ray composition, Astropart.Phys. 35 (2012) 807-812, [1203.3732].

[3] Pierre Auger Collaboration, A. Aab et al., The Pierre Auger Cosmic Ray Observatory, accepted for publication in Nucl.Instrum.Meth. A (2015) [1502.01323].

[4] Pierre Auger Collaboration, B. Kégl et al., Measurement of the muon signal using the temporal and spectral structure of the signals in surface detectors of the Pierre Auger Observatory. Proc. of the 33rd ICRC (2013), Rio de Janeiro, Brazil [1307.5059].

[5] V. Berezinsky, A. Z. Gazizov, and S. I. Grigorieva, Dip in UHECR spectrum as signature of proton interaction with CMB, Phys.Lett. B612 (2005) 147-153, [astro-ph/ 0502550 ].

[6] V. Berezinsky, A. Z. Gazizov, and S. I. Grigorieva, On astrophysical solution to ultrahigh-energy cosmic rays, Phys.Rev. D74 (2006) 043005, [hep-ph / 0204357 ].

[7] Pierre Auger Collaboration, A. Aab et al., Depth of maximum of air-shower profiles at the Pierre Auger Observatory. I. Measurements at energies above $10^{17.8}$ eV, Phys.Rev. D90 (2014) 122005, [1409.4809].

[8] Pierre Auger Collaboration, J. Abraham et al., Trigger and aperture of the surface detector array of the Pierre Auger Observatory, Nucl. Instrum. Meth. A613 (2010) 29-39.

[9] D. Heck, J. Knapp, J. N. Capdevielle, et al., CORSIKA: a Monte Carlo code to simulate extensive air showers. Forschungszentrum, Karlsruhe, 1998. FZKA 6019.

[10] S. Ostapchenko, Monte Carlo treatment of hadronic interactions in enhanced Pomeron scheme: I. QGSJET-II model, Phys.Rev. D83 (2011) 014018, [1 010 . 1869].

[11] T. Pierog, I. Karpenko, J. Katzy, E. Yatsenko, and K. Werner, EPOS LHC : test of collective hadronization with LHC data, [1306.0121]. 
[12] E.-J. Ahn, R. Engel, T. K. Gaisser, P. Lipari, and T. Stanev, Cosmic ray interaction event generator SIBYLL 2.1, Phys.Rev. D80 (2009) 094003, [0 906 . 4113].

[13] G. Battistoni, M. Garzelli, E. Gadioli, S. Muraro, P. Sala, et al., The Hadronic models for cosmic ray physics: The FLUKA code solutions, Nucl.Phys. B - Proc.Suppl. 175 (2008) 88-95, [hep-ph/0612075].

[14] S. Argiro, S. L. C. Barroso, J. Gonzalez, et al., The Offline Software Framework of the Pierre Auger Observatory, Nucl. Instrum. Meth. A580 (2007) 1485-1496, [0 707.1652 ].

[15] Pierre Auger Collaboration, A. Schulz et al., The measurement of the energy spectrum of cosmic rays above $3 \times 10^{17} \mathrm{eV}$ with the Pierre Auger Observatory. Proc. of the 33rd ICRC (2013), Rio de Janeiro, Brazil, [1307.5059].

[16] R. A. Gideon and R. A. Hollister, A rank correlation coefficient resistant to outliers, JASA 82 (1987) 656-666.

[17] E. B. Niven and C. V. Deutsch, Calculating a robust correlation coefficient and quantifying its uncertainty, Computers and Geosciences 40 (2012) 1-9.

[18] Pierre Auger Collaboration, A. Aab et al., Depth of maximum of air-shower profiles at the Pierre Auger Observatory. II. Composition implications, Phys.Rev. D90 (2014) 122006, [1 409.5083 ].

[19] Pierre Auger Collaboration, V. Verzi et al., The energy scale of the Pierre Auger Observatory. Proc. of the 33rd ICRC (2013), Rio de Janeiro, Brazil, [1307.5059].

[20] Pierre Auger Collaboration, J. Abraham et al., Measurement of the Depth of Maximum of Extensive Air Showers above $10^{18} \mathrm{eV}$, Phys. Rev. Lett. 104 (2010) 091101, [1002.0699].

[21] R. Ulrich, R. Engel, and M. Unger, Hadronic Multiparticle Production at Ultra-High Energies and Extensive Air Showers, Phys. Rev. D83 (2011) 054026, [1010.4310].

[22] T. Bergmann, R. Engel, D. Heck, et al., One-dimensional hybrid approach to extensive air shower simulation, Astropart. Phys. 26 (2007) 420-432, [astro-ph/ 0606564 ].

[23] Pierre Auger Collaboration, P. Abreu et al., Measurement of the proton-air cross-section at $\sqrt{s}=57$ TeV with the Pierre Auger Observatory, Phys.Rev.Lett. 109 (2012) 062002, [1208 . 1520 ].

[24] R. Ulrich, Extension of the measurement of the proton-air cross section with the Pierre Auger Observatory, these proceedings.

[25] Pierre Auger Collaboration, G. Farrar et al., The muon content of hybrid events recorded at the Pierre Auger Observatory. Proc. of the 33rd ICRC (2013), Rio de Janeiro, Brazil, [1307. 5059].

[26] Pierre Auger Collaboration, A. Aab et al., Muons in air showers at the Pierre Auger Observatory: Mean number in highly inclined events, Phys.Rev. D91 (2015) 032003, [1408 . 1421].

[27] L. Collica, "Measurement of the muon content in air showers at the Pierre Auger Observatory." these proceedings. 